\title{
Non-invasive detection of concomitant coronary artery anomaly and atherosclerotic coronary disease using transthoracic Doppler echocardiography
}

Barbara Uznanska-Loch, Michal Plewka, Jan Z. Peruga, Maria Krzeminska-Pakula, Jaroslaw D. Kasprzak

Chair and Department of Cardiology, Medical University of Lodz, Poland

Submitted: 29 July 2010

Accepted: 7 October 2010

Arch Med Sci 2012; 8, 1: 162-165

DOI: $10.5114 /$ aoms.2012.27296

Copyright $\odot 2012$ Termedia \& Banach

The prevalence of coronary artery anomalies (CAA) is reported to be $1 \%$ in the general population [1]. The symptoms vary from clinically silent course to angina pectoris, syncope, arrhythmias, myocardial infarction and sudden cardiac death.

Although coronary angiography remains a gold standard for the evaluation of coronary anatomy, modern echocardiography offers additional diagnostic opportunities, including assessment of proximal coronary anatomy, flow and coronary flow reserve in a variety of clinical conditions [2-6]. However, experience with echocardiography in patients with concomitant coronary anomalies and atherosclerotic diseases is rather limited.

We report a case of a 69-year-old man, with angina and ventricular arrhythmia, in whom we successfully applied echocardiographic imaging modalities to demonstrate an abnormal course of the circumflex coronary artery and identify coronary stenosis-related flow abnormalities.

A 69-year-old man was admitted to our hospital in February 2007 with chest pain on minor exertion, radiating to left arm and to the back, aggravating over the last month. Similar, less intense pain had been observed for 3 months. Concomitant history included pharmacologically controlled hypertension, type 2 diabetes mellitus with polyneuropathy, hypercholesterolaemia, peripheral artery disease, depression and benign prostatic hypertrophy.

Admission ECG was unremarkable except for a few ventricular premature beats. Admission blood pressure was 130/85.

Echocardiography revealed a minor systolic dysfunction: hypokinesis of mid- and apical segments of the lateral and posterior wall, with left ventricular ejection fraction of 58\%. The left atrium was enlarged to $45 \mathrm{~mm}$ and the left ventricular mass index was increased to $141 \mathrm{~g} / \mathrm{m}^{2}$. Mitral inflow was pseudonormal with E/A ratio 1.2. There was a mild commissural fusion of the aortic valve cusps, without significant motion impairment, a peak velocity of $1.7 \mathrm{~m} / \mathrm{s}$ and trace of regurgitation.

Serum concentrations of troponin I and CK-MB were within normal limits.

Exercise treadmill test (Bruce procedure) elicited no angina but was electrographically positive with ST segment depression in leads V4-6 at 7 METS workload. In 24-h Holter ECG monitoring 6518 ventricular premature beats but neither complex arrhythmia nor significant ST segment changes were recorded.

\author{
Corresponding author: \\ Barbara Uznanska-Loch MD \\ Chair and Department \\ of Cardiology \\ Medical University of Lodz \\ $1 / 5$ Kniaziewicza \\ 91-347 Lodz, Poland \\ Phone/fax: +48 426539909 \\ E-mail: tyhe@op.pl
}


Transthoracic echocardiography (GE, Vivid 7 Dimension; transducer M4S) of coronary arteries showed abnormal findings in the left coronary ostium, with calcifications and accelerated turbulent flow consistent with high-degree stenosis (Figure 1). Diastolic flow was accelerated to 1.3 $\mathrm{m} / \mathrm{s}$. Non-invasive Doppler transthoracic coronary flow reserve (CFR) was measured according to the standard technique, in the distal left anterior descending (LAD) coronary artery during $12 \mathrm{mg}$ adenosine injection, yielding an abnormally low value of 1.4 .

Subsequently, the abnormal coronary artery ostium was visualized in the right sinus of Valsalva, continuous with a vessel running retroaortically, consistent with the anomalous circumflex coronary artery course (Figure 2). Distal circumflex stenosis was not detectable using transthoracic echocardiography (TTE).

Coronary angiography revealed $90 \%$ ostial stenosis of the left coronary artery, with the absence of origin of the circumflex branch (CX); the distal LAD coronary artery was normal. The circumflex coronary artery originated next to the right coronary artery, running in an inferior direction, reaching its proper course after 3-4 cm; the CX was narrowed

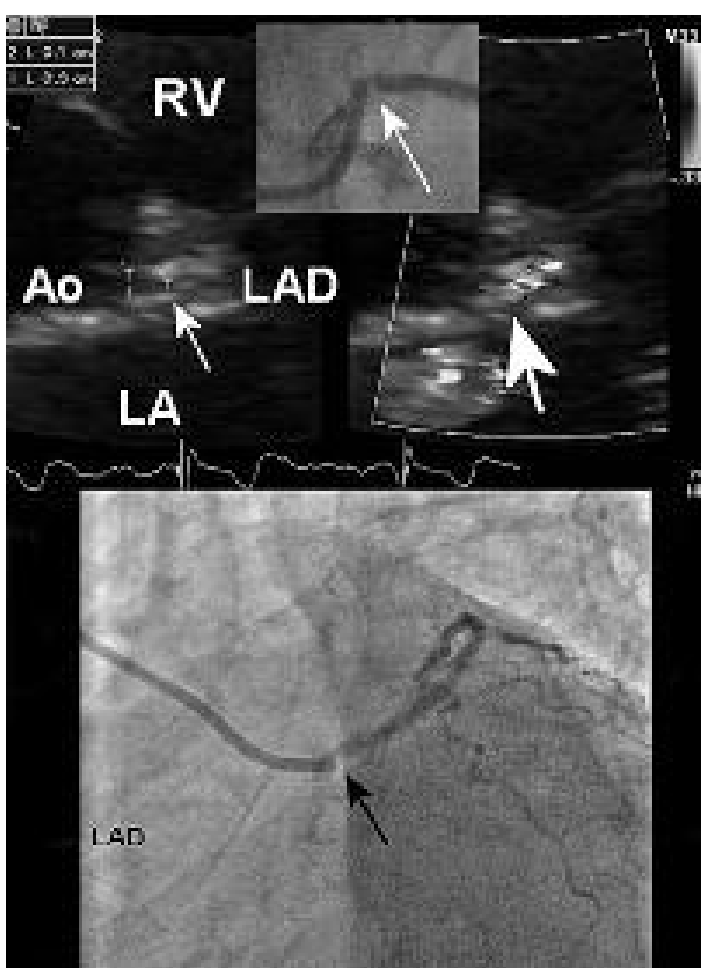

Figure 1. The arrows indicate stenosis of the left coronary ostium, detected in angiography and transtho racic echocardiography. In the panel below - normal distal left anterior descending coronary artery $L A D$ - left anterior descending coronary artery, $R V$ - right ven tricle, LA - left atrium, Ao - aorta to $90 \%$. The right coronary artery was narrow and tortuous.

The patient was referred to cardiac surgery for coronary artery bypass grafting.

Our report indicates that state-of-the-art, highend TTE allows the evaluation of coronary anatomy and coronary flow reserve, facilitating the assessment of anomalous coronary arteries combined with advanced coronary artery disease. Non-invasive data are complementary to standard angiographic findings. Angiography remains a gold standard for the evaluation of coronary anatomy. However, in some cases, echocardiography may suggest an initial diagnosis. State-of-the-art transthoracic examination is nowadays powerful enough to aid in the assessment of coronary anomalies as the ostia and proximal course can usually be visualized [7-9]. Carefully performed investigation may identify coronary disease in clinically silent or unclear cases, especially in proximal arterial segments.

Coronary artery anomalies may be clinically silent but often cause symptoms such as angina pectoris, syncope, arrhythmias, myocardial infarction, and sudden cardiac death. Importantly, coronary anomalies constitute the second most com-

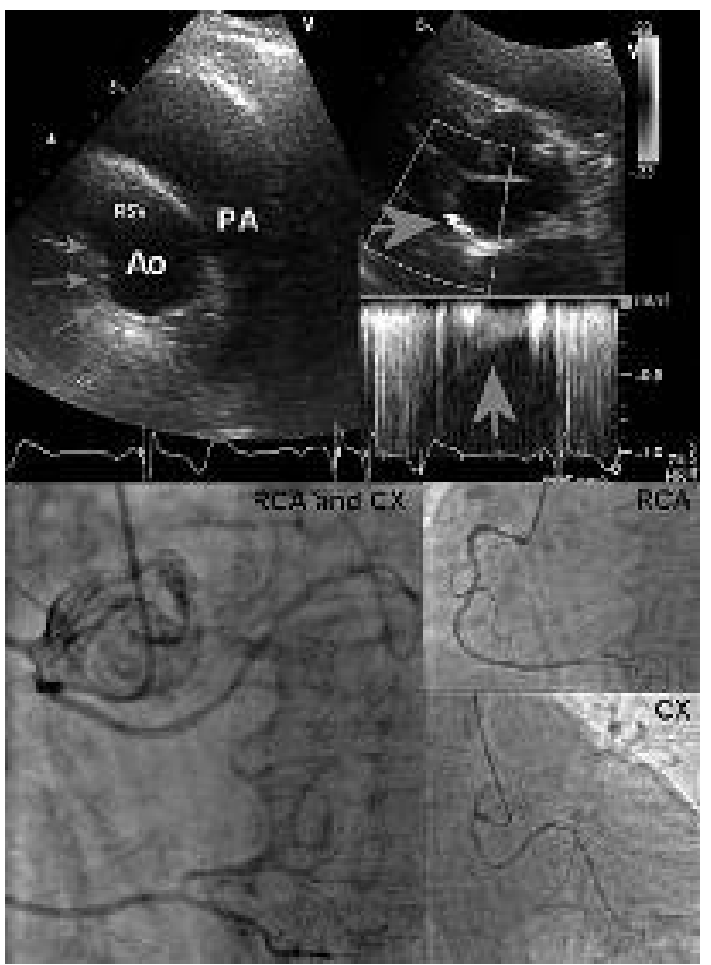

Figure 2. The arrows show anomalous circumflex coronary artery running retroaortically, in the right panels the flow detected by Doppler echocardiography. Below: angiography of the circumflex coronary artery originating next to the right coronary artery and separate angiograms of each

$R S V$ - right sinus of Valsalva, $R C A$ - right coronary artery, $P A$ - pulmonary artery, Ao - aorta, $C X$ - circumflex coronary artery 
mon cause of sudden cardiac death in young athletes. Particularly dangerous and potentially lethal variants involve Bland-White-Garland syndrome (anomalous origin of the left coronary artery from the pulmonary artery), with $80-85 \%$ mortality in the first year of life or CX coursing between the aorta and pulmonary trunk as well as the right coronary artery originating from the left sinus of Valsalva.

Circumflex branch originating from the right coronary artery (RCA) or the right sinus of Valsalva is the most common CAA, found in $0.48 \%$ of the population of patients undergoing angiography [10]. Abnormal CX may begin with a separate (37\%) or common (23\%) origin with the RCA or arise from a proximal segment of the RCA (40\%) [11]. Abnormal retroaortic segment of the vessel seems to be particularly predisposed to atherosclerosis but may be successfully treated with coronary angioplasty.

On the other hand, CX origin from non-coronary sinus is a unique anomaly, detectable by transoesophageal echocardiography, which has been reported as a useful method allowing tomographic visualization of proximal coronary arteries.

Preliminary diagnosis of coronary artery anomaly on the basis of echocardiographic examination allows one to reduce contrast and radiation exposure during angiography and thus possibly avoid some complications including contrast-induced nephropathy [12]. Patients with clinically significant type of CAA may benefit from appropriate medical or surgical treatment. Transthoracic echocardiography may be used for efficient postoperative outcome evaluation and long-term follow-up.

High-quality echocardiographic visualization of the stenotic arterial site allows quantification based on turbulent flow detection, velocity $[13,14]$ and lumen diameter measurement, and functional tests (stress echocardiography). Transthoracic examination is proven to be an accurate method to evaluate coronary artery diameter in patients with healthy coronary arteries [15]. At least doubling of maximal flow velocity within stenosis with regard to maximal flow velocity proximal to stenosis or local maximal flow velocity $>1.5 \mathrm{~m} / \mathrm{s}$ is proposed to be a reliable sign of $>50 \%$ coronary artery stenosis. When coronary angiography reveals LAD stenosis of intermediate severity, the measurement of CFR using transthoracic Doppler provides assessment of its functional importance with CFR value $>2$ justifying postponement of interventional treatment. Coronary flow reserve is thus a useful method for functional assessment of complex coronary pathophysiology.

The limitations of our report include the lack of invasive assessment of fractional flow reserve (FFR) in the LAD and CX, which would be interesting to compare with echocardiographic CFR measurements. It must be noted that distal CX stenosis was not detectable in transthoracic echocardiography.

In conclusion, coronary angiography remains a gold standard for the evaluation of coronary anatomy. Nevertheless, carefully performed transthoracic echocardiographic examination of coronary arteries may be considered as a complementary non-invasive method providing extended physiological information and enabling non-invasive follow-up in selected patients.

\section{Acknowledgments}

Source of support: Statutory Fund (Fund Number 503/1-049-01/503-01) of Chair of Cardiology, Medical University of Lodz, Lodz, Poland" .

\section{References}

1. Angelini P, Velasco JA, Flamm S. Coronary anomalies: incidence, pathophysiology, and clinical relevance. Circulation 2002; 105: 2449-54.

2. Kasprzak JD, Drozdz J, Peruga JZ, et al. Doppler detection of proximal coronary artery stenosis using transesophageal echocardiography. Kardiol Pol 1999; 50: 491-500.

3. Vrublevsky AV, Boshchenko AA, Karpov RS. Diagnostics of main coronary artery stenoses and occlusions: multiplane transoesophageal Doppler echocardiographic assessment. Eur J Echocardiogr 2001; 2: 170-7.

4. Anjaneyulu A, Raghu K, Chandramukhi S, et al. Evaluation of left main coronary artery stenosis by transthoracic echocardiography. J Am Soc Echocardiogr 2008; 21: 855-60.

5. Caiati C, Zedda N, Montaldo C, Montisci R, Iliceto S. Contrast-enhanced transthoracic second harmonic echo Doppler with adenosine: a noninvasive, rapid and effective method for coronary flow reserve assessment. J Am Coll Cardiol 1999; 34: 122-30.

6. Nemes A, Forster T, Csanády M. Coronary flow reserve in patients with aortic valve stenosis - review of the literature and own observations. Pol Przegl Kardiol 2004; 6: 203-7.

7. Kasprzak JD, Kratochwil D, Peruga JZ, et al. Coronary anomalies diagnosed with transesophageal echocardiography: complementary clinical value in adults. Int J Card Imaging 1998; 14: 89-95.

8. Fernandes F, Alam M, Smith S, Khaja F. The role of transesophageal echocardiography in identifying anomalous coronary arteries. Circulation 1993; 88: 2532-40.

9. Dawn B, Talley JD, Prince CR, et al. Two-dimensional and Doppler transesophageal echocardiographic delineation and flow characterization of anomalous coronary arteries in adults. J Am Soc Echocardiogr 2003; 16: 1274-86.

10. Wilkins CE, Betancourt B, Mathur VS, et al. Coronary artery anomalies: a review of more than 10,000 patients from The Clayton Cardiovascular Laboratories. Tex Heart Inst J 1988; 15: 166-73.

11. Leroy F, Germain S, Bauters C, Lablanche JM, Bertrand ME. Abnormal origin of the left circumflex coronary artery: clinical, angiographic and prognostic aspects. Apropos of 30 cases [French]. Arch Mal Coeur Vaiss 1992; 85: 993-9.

12. Uyarel H, Cam N, Ergelen M, et al. Contrast-induced nephropathy in patients undergoing primary angioplasty 
for acute myocardial infarction: incidence, a simple risk score, and prognosis. Arch Med Sci 2009; 5: 550-8.

13. Murata E, Hozumi T, Matsumura Y, et al. Coronary flow velocity reserve measurement in three major coronary arteries using transthoracic Doppler echocardiography. Echocardiography 2006; 23: 279-86.

14. Wierzbowska-Drabik K, Peruga Z, Plewka M, Drozdz J, Moll J, Kasprzak JD. Nonatherosclerotic ostial stenosis of left main coronary artery: echocardiographic assessment and follow-up after surgical treatment. Echocardiography 2006; 23: 133-6.

15. Kiviniemi TO, Saraste M, Koskenvuo JW, et al. Coronary artery diameter can be assessed reliably with transthoracic echocardiography. Am J Physiol Heart Circ Physiol 2004; 286: H1515-20. 